\title{
Adaptación a la población española de un instrumento de juicios morales: la batería de dilemas morales de Moore
}

\author{
Martina Carmona-Perera ${ }^{1 *}$, Alfonso Caracuel2 ${ }^{2}$ Antonio Verdejo-García ${ }^{1,3,4}$, Miguel Perez-García $1,5,6$
}

1 Departamento de personalidad, evaluación y tratamiento psicológico. Facultad de Psicología. Universidad de Granada. 2 Departamento de psicología evolutiva. Facultad de Ciencias de la Educación. Universidad de Granada. 3 Instituto de Neurociencias F. Olóriz. Centro de Investigaciones Biomédicas (CIBM). 4 School of Psychology and Psychiatry. Monash University. 5 Centro de Investigación Mente, Cerebro y Comportamiento (CIMCYC). 6 Centro de Investigación Biomédica en Red de Salud Mental (CIBERSAM).

\begin{abstract}
Resumen: Los procesos neuropsicológicos subyacentes al juicio moral son objeto de novedosas investigaciones en el ámbito de la cognición social. El objetivo de este estudio es adaptar a la población española la batería de dilemas morales de Moore. Esta batería plantea una serie de dilemas morales entre una elección utilitarista (asumir una conducta emocionalmente aversiva en favor de un beneficio mayor) y otra no-utilitarista. Participaron 154 estudiantes universitarios que completaron la versión española del instrumento. Investigamos tres tipos de dilemas: morales-personales (el propio sujeto realiza la acción aversiva, incrementando la carga emocional), morales-impersonales (la acción es indirecta, reduciendo la carga emocional), y no-morales. Las variables dependientes fueron: la proporción de respuestas afirmativas -que refleja elecciones utilitaristas, y la dificultad percibida para llevar a cabo el juicio. Los resultados mostraron buenas propiedades psicométricas y validez discriminativa en función del tipo de dilema; los dilemas morales-personales generaron menor número de elecciones utilitaristas y juicios de mayor dificultad. En conclusión, la adaptación española de cuestionario permite medir de manera fiable el juicio moral, discriminando distintos patrones de elección en función de la carga emocional, postulándose como un instrumento potencialmente útil en la evaluación de poblaciones con problemas de toma de decisiones sociales y afectivas.

Palabras clave: Juicio moral; dilemas morales; emoción; elección utilitarista.
\end{abstract}

\section{Introducción}

La Neuropsicología ha generado un interés creciente por la investigación de procesos relacionados con la cognición social, como la teoría de la mente (Grande-García, 2009; Tirapu-Ustárroz, Pérez-Sayez, Erekatxo-Bilbao y PelegrínValero, 2007; Young, Cushman, Hauser y Saxe, 2007) la empatía (Fernández-Pinto, López-Pérez y Márquez, 2008; Preston et al., 2007) y la toma de decisiones sociales y afectivas (Cortés-Pascual, 2002; Goldberg, 2001; Rilling y Sanfey, 2011; Verdejo-García y Bechara, 2010). En todos estos procesos complejos la emoción se ha revelado como un componente esencial que contribuye de manera crítica a su funcionamiento óptimo (Sánchez-Navarro y Román, 2004). En este contexto, ha surgido una nueva línea de investigación focalizada en el estudio del juicio moral, o toma de decisiones morales, y su relación con los procesos emocionales (Koenigs et al., 2007; Young, Cushman, Hauser y Saxe, 2007). La investigación científica en este ámbito se ha centrado en los juicios morales por su capacidad para guiar la conducta social de un grupo cultural, determinando así la in-

\section{* Dirección para correspondencia [Correspondence address]:}

Martina Carmona Perera. Departamento de personalidad, evaluación y tratamiento psicológico. Facultad de psicología, Campus Universitario de Cartuja, Universidad de Granada, Apdo. 18071 Granada (España). E-mail: martinacarmona@gmail.com
Title: Adaptation to Spanish population an instrument of moral judgments: Moore's battery of moral dilemmas

Abstract: The cognitive and emotional neuropsychological processes underlying moral judgments are the focus of groundbreaking research in understanding moral cognition. The aim of this study is to adapt to Spanish the moral dilemmas battery of Moore. This battery presents a set of moral dilemmas from a utilitarian choice (assuming an emotionally aversive behaviour for a higher benefit) and a non-utilitarian choice. The study involved 154 university students who were given the Spanish version of Moore's battery. We researching three types of dilemmas: moralpersonal (oneself performs the aversive action, increasing the emotional charge), impersonal-moral (the action is carried out indirectly, decreasing the emotional charge) and non-moral. The dependent variables were the proportion of affirmative answers, which reflect utilitarian choices, and the perceived difficulty to produce the judgments. The results showed good psychometric properties and significant differences in the proportion of affirmative answers and the difficulty depending on the type of dilemma, the moral-personal dilemmas generated fewer utilitarian choices and judgments with more difficulty. In conclusion, the Spanish version of Moore's battery allows measuring reliably the moral judgments, discriminating between different patterns of choice depending on the emotional charge, so it is postulated as a potentially useful instrument for assessing populations with impaired decision-making related to emotional processing.

Key words: Moral judgment; moral dilemmas; emotion; utilitarian choice.

clinación a comportarse de cierta forma (Casebeer, 2003; Greene, 2003; Moll, Zahn, Oliveira-Souza, Krueger y Grafman, 2005). El juicio moral también se denomina toma de decisiones morales y se concibe como un tipo de evaluación basada en la adecuación de la propia conducta y la de los demás a las ideas sociales de lo que es correcto y de lo que es incorrecto según el consenso de costumbres y conductas de una determinada cultura (Moll, Zahn, Oliveira-Souza, Krueger y Grafman, 2005).

En los últimos años un gran número de estudios demuestran la implicación de procesos emocionales en la toma de decisiones morales. Los estudios con pacientes con daño cerebral en regiones implicadas en la integración de contenidos cognitivos y marcadores afectivos, como la Corteza Prefrontal Ventromedial (VMPFC), han demostrado una asociación entre los déficits de procesamiento de información afectiva y las alteraciones de la toma de decisiones en situaciones morales, favoreciendo opciones de respuesta utilitaristas -donde se desconsidera el daño inflingido a una persona si éste es en beneficio del colectivo (Anderson, Bechara, Damasio, Tranel y Damasio, 1999; Greene, Sommerville, Nystrom, Darley y Cohen, 2001; Greene, Nystrom, Engell, Darley y Cohen, 2004; Koenigs et al., 2007). Los estudios de neuroimagen en voluntarios sanos corroboran el papel central del procesamiento afectivo sobre los juicios morales, demostrando la activación de regiones implicadas en el pro- 
cesamiento emocional (p.e., corteza prefrontal ventromedial, ínsula y amígdala) durante la consideración de los dilemas morales (Heekeren, Wartenburger, Schmidt, Schwintowski y Villringer, 2005; Luo y Blair, 2006; Oliveira-Souza, Bramati y Grafman, 2002; Young y Koenigs, 2007).

La metodología utilizada en el estudio neuropsicológico de la cognición moral ha generado distintos tipos de instrumentos, incluyendo los que se basan en la presentación de imágenes morales (Moll, Oliveira-Souza, Bramati y Grafman, 2002; Vélez, Chayo, Arias, Castillo y Ostrosky, 2003), la lectura de enunciados con contenido moral (Heekeren et al., 2005; Moll, Oliverira-Souza, Moll e Ignacio, 2005) o la asociación de contenidos morales (Nosek, Banaji y Greenwald, 2002). Sin embargo, la metodología más aceptada consiste en el uso de dilemas morales, descritos textualmente, en los que el sujeto debe decidir entre una opción utilitarista (dañar a alguien en beneficio del colectivo) y una opción no utilitarista (renunciar a dañar a alguien incluso en perjuicio del bien global). Esta metodología ha dado lugar a diversos cuestionarios, entre los que destaca la batería de dilemas morales de Moore (Moore, Clark y Kane, 2008) que supera algunas de las limitaciones de otros instrumentos (p.e., Greene et al., 2001), incluyendo las diferencias de longitud entre dilemas, los diferentes grados de abstracción, o la contaminación de otros procesos subyacentes, como la memoria de trabajo o el razonamiento probabilístico (Hauser, Cushman, Young, Jin y Mikhail, 2007; Moore et al., 2008).

La batería de dilemas morales de Moore está formada por 38 escenarios hipotéticos sobre los que el sujeto debe realizar juicios dicotómicos acerca de cómo se comportaría si se enfrentara a ese dilema, aceptando o rechazando la acción que les propone el dilema. Las respuestas afirmativas se conciben como respuestas utilitaristas, ya que en ellas el sujeto acepta llevar a cabo una acción emocionalmente muy aversiva a favor del bienestar agregado; por ejemplo, arrojar al mar a un moribundo para mantener a flote un bote salvavidas. En cambio, en las respuestas negativas el sujeto asume una elección no utilitarista, en contra de lo ventajoso en términos de coste-beneficio; por ejemplo, decidir no arrojar al moribundo pese a que esto pone en riesgo la vida propia y las del grupo. Complementando la propuesta de Greene et al. (2001), que distinguieron entre dilemas personales (matar directamente a una persona para salvar la vida de los demás) e impersonales (llevar a cabo acciones que de forma incidental o secundaria matan a la persona), Moore et al. añaden dos tipologías más: (i) autobeneficio (corre peligro la propia vida) vs. beneficio ajeno (corre peligro la vida de los demás) y (ii) muerte inevitable (se muere una persona independientemente de la acción del sujeto) vs. muerte evitable (se muere una persona solo si el sujeto actúa). Esta nueva tipología permite estudiar de manera más precisa la influencia de variables como la carga emocional, el beneficio para el agente y la posibilidad de evitar o no víctimas mortales como resultado de nuestra acción.

Los resultados del análisis de la batería original de Moore (Moore et al., 2008) muestran una menor proporción de res- puestas afirmativas en los dilemas personales (con mayor carga emocional) que en los impersonales. Además, en los dilemas personales los participantes tardan más en decidirse, mostrando mayores tiempos de reacción que en los dilemas impersonales. Este incremento de los tiempos de reacción es considerado un indicador del grado de conflicto emocional o dificultad generada por el dilema (Greene et., al 2001; 2004). Aunque los estudios originales no han evaluado explícitamente esta dimensión, otros estudios (Koenigs et al 2007; Velez-García et al., 2009) han utilizado índices de la dificultad subjetiva del juicio a través de escalas Likert entre 7 y 10 valores. Siguiendo esta línea de trabajo, hemos incorporado una medida de dificultad percibida generada por el juicio, con el objetivo de validar la distinción entre dilemas personales e impersonales criticada por algunos autores por basarse simplemente en las respuestas conductuales, sin tener en cuenta medidas subjetivas (Kahane y Shackel, 2008; McGuire et al., 2009). Por otro lado, la decisión de "matar para salvar la vida de uno mismo y la de los demás" (dilema de autobeneficio) es más frecuente que la de "matar para salvar sólo otras vidas" (dilema de beneficio ajeno). Respecto la variable muerte evitable-muerte inevitable, los participantes tienden a elegir "matar a alguien cuya muerte es inevitable" que si no lo es. Según varios autores (Greene et al., 2004, 2007; Koenigs et al., 2007; Moore et al., 2008) estos resultados se ajustan a las predicciones de la teoría del doble proceso (Greene et al., 2001). Esta teoría postula que en el transcurso de la toma de decisiones morales se produce un conflicto entre mecanismos emocionales y cognitivos, de manera que cuando la cognición supera la emoción el resultado sería una respuesta utilitarista que maximiza el bienestar agregado; sin embargo, cuando predomina la emoción sobre la razón, se responde en contra de infligir el daño.

En función de estos resultados previos, nuestro objetivo es generar una versión española de la batería de dilemas morales de Moore (2008), y comprobar si la tipología propuesta por este autor (dilemas personales $v s$. impersonales, autobeneficio $v$ s. beneficio ajeno y muerte evitable $v s$. muerte inevitable), que refleja distintos grados de carga e implicación emocional, permite discriminar distintas tendencias de decisión en nuestra cultura. Nuestras predicciones son: (i) que la versión española de la batería, en la que incluimos una nueva medida de dificultad de juicio para tomar la decisión moral, presentará adecuadas propiedades psicométricas y equivalencia con la versión original, y (ii) que -de acuerdo con la teoría del doble proceso, los dilemas con mayor carga e implicación emocional generarán mayor dificultad de juicio y menor número de decisiones utilitaristas.

\section{Método}

\section{Participantes}

Participaron 154 estudiantes de grado de la Universidad de Granada, 120 mujeres y 34 hombres. La edad promedio de los sujetos fue de 21.51 años, con un rango entre 18 y 54 
años. La variable nivel educativo varía entre los 15 y los 27 años de formación académica, siendo el promedio de dieciséis años y ocho meses.

\section{Instrumentos}

Batería de dilemas morales de Moore adaptada a la población española:

La adaptación del cuestionario se llevó a cabo mediante un procedimiento estandarizado de traducción, retrotraducción y revisión de la equivalencia lingüística y conceptual entre las versiones original y retro-traducida por un comité de expertos en neuropsicología transcultural.

Siguiendo los parámetros del cuestionario original de Moore (2008) la versión española también se compone de dos formas paralelas: A y B. Cada una de ellas está formada por 2 dilemas de prueba, 12 dilemas no morales y 24 dilemas morales que suman un total de 38 escenarios hipotéticos. Los dilemas no morales son ítems de control que se utilizan para garantizar la comprensión lectora y capacidad de razonamiento de las personas evaluadas. Para la construcción de las formas paralelas, los dilemas morales mantienen su contenido o tema central, pero cambia la redacción de algunos detalles para modificar la dimensión personal-impersonal, de modo que los ítems de la categoría personal de la forma A se conviertan en impersonales en la forma B, y al contrario. Los escenarios morales se dividen en cuatro tipologías: autobeneficio-muerte inevitable, autobeneficio-muerte evitable, beneficio ajeno-muerte inevitable y beneficio ajeno-muerte evitable. Para cada una de ellas, el cuestionario incluye tres dilemas personales y tres dilemas impersonales. Combinando las categorías anteriores se obtienen ocho tipologías de dilemas morales: 1) Autobeneficio muerte evitable e impersonal (Yo-Evitable-Impersonal); 2) Autobeneficio muerte evitable y personal (Yo-Evitable-Personal); 3) Autobeneficio muerte inevitable e impersonal (Yo-Inevitable-Impersonal); 4) Autobeneficio muerte inevitable y personal (YoInevitable-Personal); 5) Beneficio ajeno muerte evitable e impersonal (Otro-Evitable-Impersonal); 6) Beneficio ajeno muerte evitable y personal (Otro-Evitable-Personal); 7) Beneficio ajeno muerte inevitable e impersonal (OtroInevitable-Impersonal); 8) Beneficio ajeno muerte inevitable y personal (Otro-Inevitable-Personal). Los sujetos responden ante todos los dilemas de forma dicotómica, "sî" o "no". Con el objetivo de la validar la distinción entre los dilemas, en la adaptación del cuestionario hemos añadido una medida de dificultad de juicio mediante una escala Likert del 1 al 10 dónde el sujeto evalúa la dificultad para emitir el juicio en cada uno de los dilemas.

\section{Procedimiento}

La selección de los sujetos se llevó a cabo de forma aleatoria entre varias facultades de la Universidad de Granada. Todos los participantes eran informados sobre las características del estudio y accedieron a participar de forma volunta- ria. Los sujetos eran evaluados de forma colectiva en grupos de 20 personas y el tiempo de duración de la misma solía oscilar entre 45 y 60 minutos; no se estableció un límite de tiempo para su cumplimentación. La administración de la prueba se realizó en papel y lápiz y en presencia de un experimentador instruido en la tarea de dilemas morales que controló su correcta realización y solventó las dudas de los participantes.

\section{Variables y análisis estadísticos}

Las variables dependientes utilizadas en los análisis estadísticos fueron los siguientes:

- Respuestas afirmativas (RA): Es la proporción de respuestas "sî" que emiten los sujetos ante todos los dilemas, la opción de respuesta "sí" significa que aceptan llevar a cabo la acción que les propone el dilema, y en el caso de los dilemas morales se corresponde siempre con la opción utilitarista (Moore et al., 2008).

- Dificultad de juicio (DJ): Es la media del índice de dificultad percibida para cada tipo de dilemas. El índice de dificultad de juicio evalúa la dificultad para emitir cada uno de los juicios que lleva a cabo el sujeto y oscila del 1 , dificultad de juicio muy baja, al 10, máxima dificultad de juicio.

Para determinar las propiedades psicométricas del cuestionario se aplicaron análisis estadísticos tanto del Modelo Lineal General como de la Teoría de Respuesta al Ítem (TRI). De los modelos dentro de la TRI hemos aplicado el modelo de Rasch, que de una forma sencilla y robusta permite el estudio psicométrico de instrumentos para la medida de comportamientos, actitudes y percepciones (Bond y Fox, 2007). En primer lugar se llevó a cabo un análisis de Rasch para determinar la unidimensionalidad del cuestionario y la calidad de los ítems. A continuación, para la fiabilidad se utilizó el coeficiente alfa de Cronbach y la correlación entre dos mitades, así como el Índice de Separación de Personas del análisis de Rasch. Para examinar la estructura del cuestionario se llevó a cabo un análisis factorial exploratorio utilizando el método de análisis de componentes principales. Se aplicó una prueba $t$ de Student para muestras independientes para examinar la equivalencia de las formas A y B. Una vez confirmada esta equivalencia, los resultados de ambas formas se agruparon para llevar a cabo el resto de análisis estadísticos. La validez discriminativa de los índices de RA y DJ, en función del factor Tipo de Dilema (8 tipos), se analizó mediante dos ANOVAs de medidas repetidas con tres factores de dos niveles cada uno: Personal-Impersonal, Evitable-Inevitable y Autobeneficio-Beneficio Ajeno. Los efectos de interacción se analizaron mediante comparaciones pareadas usando pruebas $t$ de Student. Finalmente, llevamos a cabo un análisis de probabilidad binomial para comparar los resultados de la versión española con los obtenidos por Moore (2008) utilizando el instrumento original. Los análisis se han realizado con los programas SPSS (IBM SPSS Statis- 
tics, Somers, New York) y RUMM2030 (RUMM Laboratory Pty Ltd, Perth, Australia).

\section{Resultados}

\section{Propiedades psicométricas}

\section{Unidimensionalidad}

Se han llevado a cabo sendos análisis de Rasch para los ítems morales de cada una de las formas del cuestionario. En cada análisis los ítems se han agrupado en 8 subgrupos de acuerdo a las tipologías que propone el autor. En primer lugar se ha comprobado el ajuste al modelo de Rasch considerando el estadístico ítem-rasgo y a continuación se ha comprobado si el conjunto de ítems morales es unidimensional mediante el método más recomendado y exigente dentro de este modelo (Tennant y Pallant, 2006), que consiste en realizar análisis de componentes principales de los residuos, definiendo 2 subgrupos de ítems según la carga positiva o negativa de cada ítem en el primer componente (Smith y Miao, 1994). Se comprueba el ajuste al modelo de Rasch de cada subset por separado y se obtiene la estimación de la localización de los sujetos en cada uno y posteriormente son comparadas mediante una prueba $t$ apareada. El criterio utilizado para aceptar o rechazar la unidimensionalidad es que el porcentaje de $t$ tests que quedan fuera del intervalo de confianza al $95 \%$ no exceda el 5\% del total (Tennant y Conaghan, 2007)

Los resultados no significativos del estadístico ji cuadrado en ambas formas indican un buen ajuste al modelo de Rasch (forma A: $x^{2}=5.29, p=.72$; forma B: $x^{2}=13.05, p$ $=.11$ ). La unidimensionalidad de la forma A queda ampliamente demostrada por un porcentaje de $t$ tests significativos de solo el $1.27 \%$. Sin embargo, en la forma B la demostración es más limitada, ya que supera de manera muy leve el criterio establecido $(5.56 \%)$.

En cuanto a la calidad de los ítems, se han aplicado los criterios de bondad de ajuste de cada ítem al modelo de Rasch. Este análisis se realiza mediante la estandarización de los residuos de las respuestas de todas las personas a cada ítem, estableciendo como criterio que el ajuste al modelo es aceptable cuando los residuos están dentro del rango \pm 2.5 y tienen un valor de ji cuadrado no significativo tras el ajuste de Bonferroni (Tennant y Pallant, 2006). La falta de ajuste al modelo de Rasch indicaría que el ítem no contribuye al constructo medido, en este caso la toma de decisiones morales. En ambas formas del cuestionario, todos los ítems cumplen el criterio de bondad de ajuste, por lo tanto contribuyen al estudio del constructo latente. También se ha comprobado que ninguno de los dilemas presenta funcionamiento diferencial del ítem (DIF) para los distintos grupos de partici- pantes (hombres y mujeres y los grupos que el software organiza en función de la cantidad de constructo latente).

\section{Fiabilidad}

En la variable RA se obtuvo un alfa de Cronbach de 0.89 para la forma A y de 0.87 para la forma B. El análisis de la fiabilidad por el procedimiento de dos mitades mostró un coeficiente de Spearman-Brown de 0.87 para la forma A y de 0.88 para la forma B. También se obtuvo una alta consistencia interna (forma $\mathrm{A}=0.95 \mathrm{y}$ forma $\mathrm{B}=0.94$ ) y una alta fiabilidad de dos mitades (forma $\mathrm{A}=0.86$ y forma $\mathrm{B}=0.94$ ) para la variable DJ.

Usando análisis Rash, se ha obtenido un Índice de Separación de Personas (PSI; Person Separation Index) de 0.86 para la forma A y de 0.83 para la forma B. Este índice se interpreta de forma análoga al índice de fiabilidad alfa de Cronbach y se considera aceptable a partir de 0.8 (Fisher, 1992).

\section{Estructura Interna}

Se ha realizado un análisis factorial exploratorio para investigar la estructura dimensional de los dilemas morales en las formas A y B del cuestionario. Con el método de componentes principales y la rotación Varimax basada en autovalores mayores de $1 \mathrm{y}$ de acuerdo al gráfico de sedimentación, en ambas formas la solución más ajustada corresponde a seis factores, con una varianza común explicada de 60.38 $\%$ para la forma A y $62.24 \%$ para la forma B. La interpretación de seis factores no se corresponde con las dimensiones teóricas propuestas por el autor, por lo que a continuación se llevó a cabo un análisis factorial forzando la extracción de dos factores en un intento de validación de la dimensión principal Personal-Impersonal o de alguna de las otras dos (beneficio propio-ajeno, muerte evitable-inevitable). Tras la rotación Varimax, el primer factor explicó el $19.16 \%$ en la forma $\mathrm{A}$ y el $21.48 \%$ en la $\mathrm{B}$ y el segundo factor un $17.63 \%$ y $16.77 \%$ respectivamente. El ajuste muestral se confirmó con un valor del índice Kaiser-Meyer-Olkin de 0.77 y 0.80 y un test de esfericidad de Bartlett significativo en ambas formas.

En cuanto a la correspondencia de los ítems con alguna de las dimensiones dicotómicas propuestas por Moore, la dimensión personal o impersonal es la que permite una mejor interpretación de los factores porque la mayoría de los ítems de cada categoría presentan una carga mayor en el mismo factor. En la forma A, 17 de los 24 ítems se agruparon en el factor correspondiente, mientras que en la forma $\mathrm{B}$ 20 de los 24 (ver Tabla 1). En cuanto a los ítems que presentan autovalores inferiores a 0.4 , en la forma A solo hay dos, sin embargo, en la forma $\mathrm{B}$ el número aumenta hasta cinco. 
Tabla 1. Cargas factoriales en la solución de dos factores en las formas A y B.

\begin{tabular}{|c|c|c|c|c|c|}
\hline \multirow{3}{*}{$\begin{array}{l}\overline{\text { Forma A }} \\
\text { Dilema }\end{array}$} & & & \multicolumn{3}{|l|}{ Forma B } \\
\hline & \multicolumn{2}{|c|}{ Componente } & \multirow[t]{2}{*}{ Dilema } & \multicolumn{2}{|c|}{ Componente } \\
\hline & $1 \mathrm{Imp}$ & 2 Pers & & $1 \mathrm{Imp}$ & 2 Pers \\
\hline Reactor nuclear (Yo_inevit_pers) & 0.725 & & Humos (Otro_inevit_imp) & 0.721 & \\
\hline Espía enemigo (Otro_inevit_imp) & 0.643 & & Reactor nuclear (Yo_inevit_imp) & 0.718 & \\
\hline Prevenir ébola (Yo_evit_imp) & 0.570 & & Estación espacial(Yo_inevit_imp) & 0.673 & \\
\hline Bloque piedra (Yo_inevit_imp) & 0.556 & & Rescate 911 (Yo_inevit_imp) & 0.637 & \\
\hline Autobús (Yo_inevit_imp) & 0.488 & & Submarino (Yo_evit_imp) & 0.585 & \\
\hline Orfanato (Yo_evit_imp) & 0.453 & & Bote (Otro_inevit_imp) & 0.481 & \\
\hline Muelles (Otro_evit_pers) & 0.542 & & Llanto bebé (Yo_evit_imp) & 0.428 & \\
\hline Ataque tiburón (Yo_evit_imp) & 0.444 & & Edificio en llamas (Yo_evit_imp) & 0.336 & \\
\hline Premio nobel (Otro_evit_imp) & 0.253 & & Premio nobel (Otro_evit_pers) & 0.290 & \\
\hline Edificio en llamas (Yo_evit_pers) & & 0.742 & Muelles (Otro_evit_imp) & 0.283 & \\
\hline Suspenso (Yo_inevit_imp) & & 0.639 & Orfanato (Yo_evit_pers) & & 0.762 \\
\hline Estación espacial (Yo_inevit_pers) & & 0.611 & Tren (Otro_evit_pers) & & 0.702 \\
\hline Motocicleta (Otro_evit_imp) & & 0.608 & Suspenso (Yo_inevit_pers) & & 0.677 \\
\hline Humos (Otro_inevit_pers) & & 0.588 & Bloque piedra (Yo_inevit_pers) & & 0.593 \\
\hline Pozo de mina (Otro_inevit_imp) & & 0.586 & El magnate (Otro_inevit_pers) & & 0.493 \\
\hline El magnate (Otro_inevit_imp) & & 0.531 & Ataque tiburón (Yo_evit_pers) & & 0.486 \\
\hline Trasplante (Otro_evit_pers) & & 0.520 & Espía enemigo(Otro_inevit_pers) & & 0.483 \\
\hline Vacuna (Otro_evit_pers) & & 0.514 & Pozo de mina (Otro_inevit_pers) & & 0.476 \\
\hline Submarino (Yo_evit_pers) & & 0.510 & Autobús (Yo_inevit_pers) & & 0.468 \\
\hline Tren (Otro_evit_imp) & & 0.499 & Prevenir ébola (Yo_evit_pers) & & 0.426 \\
\hline Rescate 911 (Yo_inevit_pers) & & 0.474 & Motocicleta (Otro_evit_pers) & & 0.424 \\
\hline Llanto Bebé (Yo_evit_pers) & & 0.451 & Transplante (Otro_evit_imp) & & 0.418 \\
\hline Bote (Otro_inevit_pers) & & 0.417 & Vacuna (Otro_evit_imp) & & 0.382 \\
\hline Eutanasia (Otro_inevit_pers) & & 0.378 & Eutanasia (Otro_inevit_imp) & & 0.326 \\
\hline
\end{tabular}

Nota: La abreviatura Imp. indica dilemas de bajo conflicto emocional; Pers. se refiere a los dilemas de alto conflicto emocional; Yo indica dilemas que implican autobeneficio; Otro indica dilemas que implican beneficio ajeno; Evit. indica dilemas dónde la muerte es evitable; Inevit. se refiere a los dilemas en que la muerte es inevitable.

Tabla 2. Análisis entre grupos para comprobar la equivalencia de la forma A y la forma B de la adaptación española del cuestionario de Moore.

\begin{tabular}{|c|c|c|c|c|c|}
\hline Variable dependiente & Tipo de dilema & $\begin{array}{l}\text { Media (DT) } \\
\text { Moore A }\end{array}$ & $\begin{array}{l}\text { Media (DT) } \\
\text { Moore B }\end{array}$ & $t$ & $p$ \\
\hline Proporción de respuestas afirmativas & No moral & $61.18(14.30)$ & $58.56(14.22)$ & 1.120 & .264 \\
\hline \multirow{8}{*}{$(\mathrm{RA})$} & Yo_evitable_impersonal & $61.66(33.58)$ & $64.81(24.94)$ & -0.650 & .517 \\
\hline & Yo_evitable_personal & $43.58(30.53)$ & $46.29(34.28)$ & -0.511 & .610 \\
\hline & Yo_inevitable_impersonal & $54.70(36.81)$ & $80.82(28.82)$ & -4.832 & .000 \\
\hline & Yo_inevitable_personal & $61.60(36.62)$ & $56.62(39.94)$ & 0.802 & .424 \\
\hline & Otro_evitable_impersonal & $57.08(31.01)$ & $52.31(26.13)$ & 1.019 & .310 \\
\hline & Otro_evitable_personal & $45.99(31.71)$ & $31.48(30.06)$ & 2.878 & .005 \\
\hline & Otro_inevitable_impersonal & $51.05(23.16)$ & $81.48(25.56)$ & -7.672 & .000 \\
\hline & Otro_inevitable_personal & $41.45(37.29)$ & $44.44(29.60)$ & -0.541 & .589 \\
\hline \multirow[t]{9}{*}{ Media de la dificultad de juicio (DJ) } & No moral & $3.40(1.28)$ & $3.54(1.42)$ & -0.626 & .533 \\
\hline & Yo_evitable_impersonal & $6.71(2.32)$ & $7.37(2.26)$ & -1.743 & .084 \\
\hline & Yo_evitable_personal & $6.16(2.39)$ & $6.97(2.41)$ & -2.029 & .044 \\
\hline & Yo_inevitable_impersonal & $7.37(2.28)$ & $7.55(2.24)$ & -0.464 & .640 \\
\hline & Yo_inevitable_personal & $7.25(2.47)$ & $7.78(2.13)$ & -1.359 & .176 \\
\hline & Otrotro_evitable_impersonal & $6.54(2.09)$ & $6.59(2.19)$ & -0.152 & .880 \\
\hline & Otro_evitable_personal & $6.11(2.26)$ & $6.40(2.44)$ & -0.741 & .560 \\
\hline & Otro_inevitable_impersonal & $5.57(2.16)$ & $7.34(2.00)$ & -5.175 & .000 \\
\hline & Otro_inevitable_personal & $6.77(2.33)$ & $6.23(2.27)$ & 1.436 & .153 \\
\hline
\end{tabular}

Nota: La abreviatura Yo, indica dilemas que implican autobeneficio; Otros indica dilemas que implican beneficio ajeno; Evitable indica dilemas dónde la muerte es evitable; Inevitable se refiere a los dilemas en que la muerte es inevitable: Impersonal: indica dilemas de bajo conflicto emocional; Personal se refiere a los dilemas de alto conflicto emocional.

\section{Equivalencia de las formas}

Los resultados mostraron que las dos formas del cuestionario se pueden considerar paralelas respecto a la proporción de RA, con la excepción de tres tipos de dilemas: Yo-
Inevitable-Impersonal, Otro-Evitable-Personal y OtroInevitable-Impersonal. Cuando analizamos la variable DJ las dos formas resultan equivalentes en la mayoría de las condiciones, con la excepción de los dilemas Yo-EvitablePersonal y Otro-Inevitable-Impersonal (ver Tabla 2). En 
función de estos resultados, que indican equivalencia de las formas en la mayoría de las condiciones consistentemente para ambas variables dependientes, decidimos agrupar los datos de las formas A y B en los análisis sucesivos.

\section{Diferencias entre tipos de dilemas morales según las respuestas afirmativas}

Los resultados utilizando la proporción de RA (decisiones utilitaristas) mostró que existían diferencias entre los tres principales tipos de dilemas: Personal vs. Impersonal $[F$ $(1,67)$ 84.790; $p<$.000]; Autobeneficio vs. Beneficio Ajeno $[F(1,67) 26.813 ; p<.000]$; Muerte evitable vs. Muerte inevitable $[F(1,67) 29.944 ; p<.000]$, así como una interacción entre las tres variables $[F(1,67) 12.646 ; p=.001]$. Las comparaciones pareadas mostraron efectos significativos de la mayoría de los contrastes: los dilemas Impersonales, de Muerte Inevitable y de Autobeneficio obtenían proporciones de RA significativamente mayores (Tabla 3, panel superior).

Tabla 3. Análisis de varianza y comparaciones post-hoc realizadas con un ANOVA y una T de medidas repetidas respectivamente. Adaptación española de la batería de Moore.

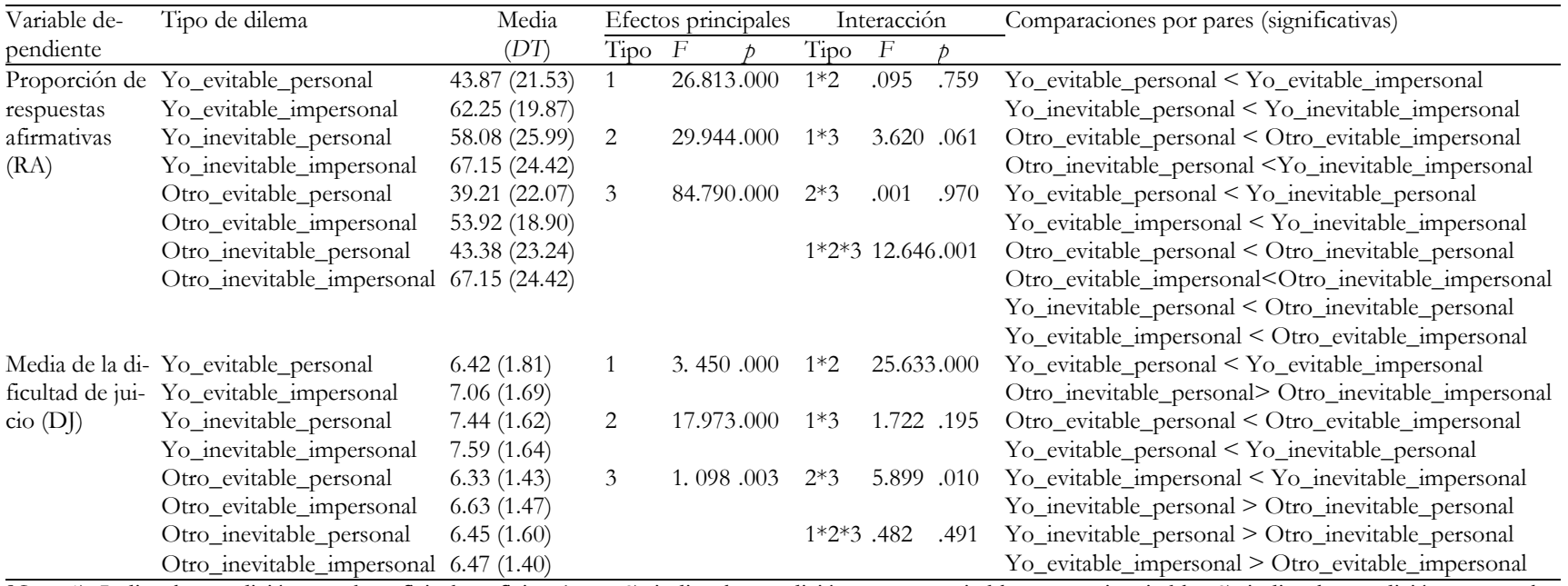

Nota: 1) Indica la condición autobeneficio-beneficio ajeno; 2) indica la condición muerte evitable-muerte inevitable; 3) indica la condición personalimpersonal. La abreviatura Yo, indica dilemas que implican autobeneficio; Otro indica dilemas que implican beneficio ajeno; Evitable indica dilemas dónde la muerte es evitable; Inevitable se refiere a los dilemas en que la muerte es inevitable: Impersonal indica dilemas de bajo conflicto emocional; Personal se refiere a los dilemas de alto conflicto emocional.

\section{Dificultad de juicio en cada tipo de dilema moral}

Los resultados mostraron diferencias en los efectos principales Personal-Impersonal [ $F(1,51) 1.098$; $p=.003$ ], AutobeneFicio-BeneFicio Ajeno [F (1,51) 3.450; $p<.000]$ y Muerte Evitable-Muerte Inevitable [F (1,51) $17.973 ; p<$ .000]. Las interacciones de segundo orden Fueron significativas en las condiciones de Personal/Impersonal vs. Evitable/Inevitable $[F(1,51) 5.899 ; p=.010]$ y de AutobeneFicio/BeneFicio Ajeno vs. Evitable/Inevitable $[F(1,51)$ 25.633; $p<.000]$. Las comparaciones pareadas mostraron efectos significativos en dos de los cuatro pares de la interacción Personal vs. Impersonal (Autobeneficio-Muerte Evitable y Beneficio Ajeno-Muerte Inevitable), indicando en ambos casos que los dilemas personales presentan mayor dificultad de juicio que los dilemas impersonales. Los dilemas de Autobeneficio generaron mayor dificultad de juicio que los de Beneficio Ajeno, con independencia de la implicación personal y la evitabilidad. En las condiciones de Autobeneficio Personal e Impersonal las muertes Inevitables generaron mayor dificultad de juicio (Tabla 3, panel inferior).

La distribución de las puntuaciones descriptivas de la dificultad de juicio (Ver Figura 1) muestra un mayor porcen- taje de respuestas de las categorías más extremas 1 y 10 que indican que responder al dilema les ha resultado muy fácil o muy difícil, mientras que las opciones intermedias obtienen porcentajes de respuesta muy similares.

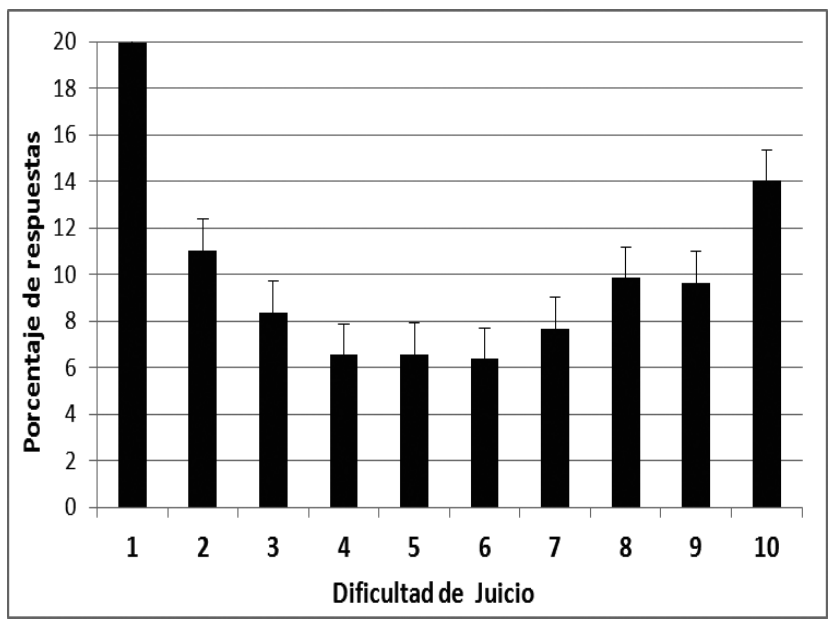

Figura 1. Distribución de las puntuaciones descriptivas de la dificultad de juicio, indicando el porcentaje de respuestas de cada categoría de la escala Likert "Dificultad de Juicio". 
Comparación entre el cuestionario original de Moore (2008) y su adaptación a la población Española

Las proporciones de RA en función del tipo de dilemas morales respecto el efecto principal Personal-Impersonal, fueron muy similares (ver Figura 2). El análisis de probabilidades binomiales comparando la proporción de RA para cada tipo de dilema mostró que no existían diferencias significativas entre los dos instrumentos $(\phi>.05$, ver Tabla 4). Por tanto, la distinción entre los distintos tipos de dilemas morales encontradas en la adaptación española se corresponde con la del cuestionario original de Moore, dónde se distinguen ocho tipos de dilemas morales.
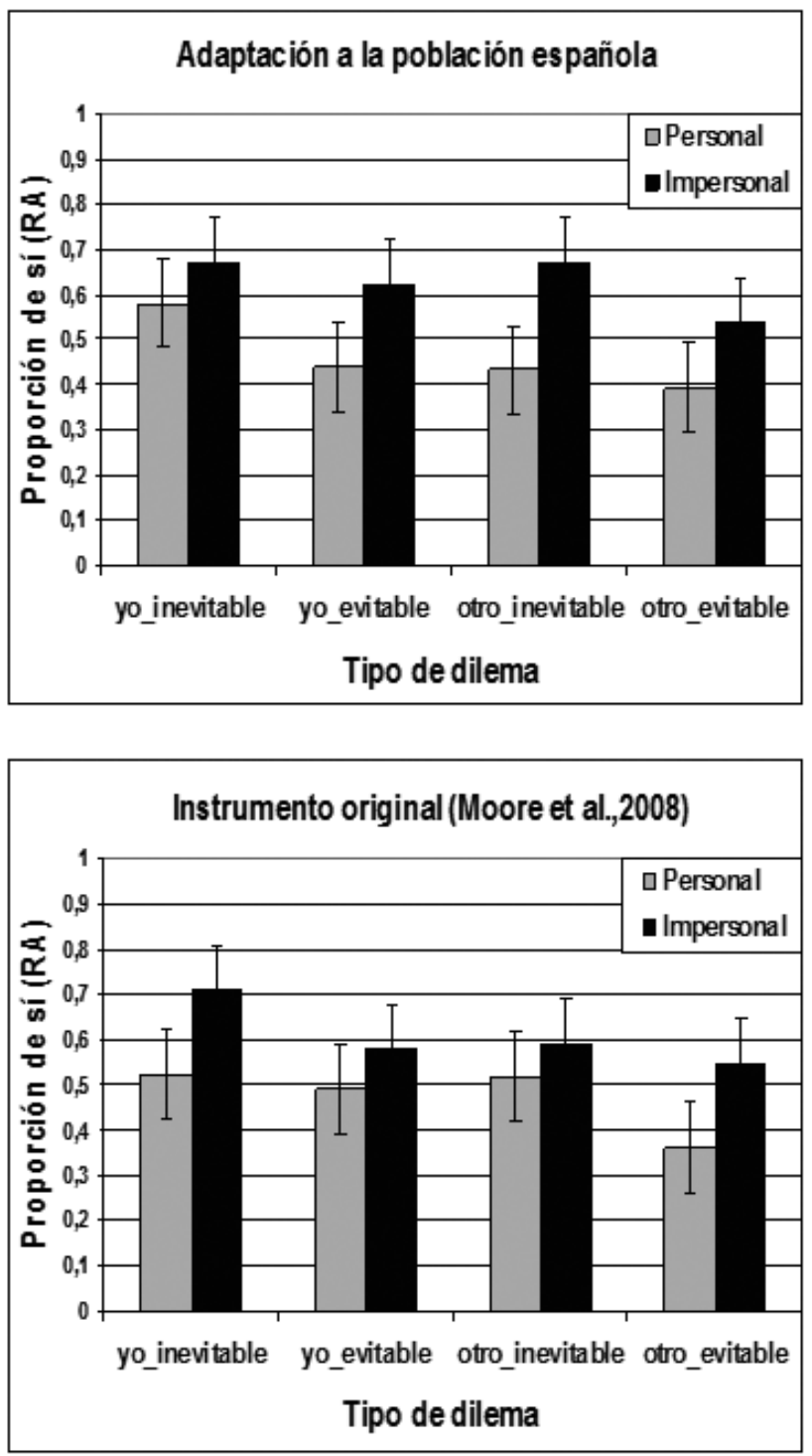

Figura 2. Proporción de respuestas afirmativas en función del tipo de dilema moral. Comparación entre los resultados de la batería original de Moore (2008) con los hallados en la adaptación española del instrumento.
Tabla 4. Análisis de Probabilidad en la variable dependiente proporción de Sí (RA). Comparación entre los resultados de la batería original de Moore (2008) con los hallados en la alaptacionn española del instrumento.

\begin{tabular}{lccc}
\hline Tipo de dilema & $\begin{array}{c}\text { Batería Original } \\
\text { de Moore (2008) } \\
\text { Proporción } \\
\text { de Sí (RA) }\end{array}$ & $\begin{array}{c}\text { Adaptación } \\
\text { Española de } \\
\text { Moore. Propor- } \\
\text { ción de Sí (RA) }\end{array}$ & $p$ \\
\hline Yo_evitable_personal & .43 & .49 & .313 \\
Yo_evitable_impersonal & .62 & .58 & .494 \\
Yo_inevitable_personal & .58 & .52 & .366 \\
Yo_inevitable_impersonal & .67 & .71 & .442 \\
Otro_evitable_personal & .39 & .36 & .616 \\
Otro_evitable_impersonal & .53 & .55 & .706 \\
Otro_inevitable_personal & .43 & .52 & .126 \\
Otro_inevitable_impersonal & .67 & .59 & .188 \\
\hline
\end{tabular}

\section{Discusión}

Este estudio proporciona tres hallazgos principales: (i) la adaptación española de la batería de dilemas de Moore et al. (2008) presenta propiedades psicométricas adecuadas y sus resultados son equivalentes a los obtenidos con la versión original; (ii) los resultados de la variable "proporción de respuestas afirmativas" replicaron los efectos discriminativos propuestos en la versión original: los dilemas personales, de muerte evitable y que no reportan autobeneficio generan un menor número de respuestas utilitaristas; y (iii) los resultados de la variable "dificultad de juicio" son congruentes con el grado de conflicto emocional de las decisiones morales: las decisiones personales, de muerte inevitable y de autobeneficio generaron una mayor dificultad de juicio.

Los buenos datos psicométricos, en términos de unidimensionalidad, fiabilidad y validez discriminativa de los tipos de dilemas, así como la equivalencia con los resultados del cuestionario original, postulan esta versión de la batería de dilemas de Moore et al. (2008) como un instrumento potencialmente útil para la investigación de fenómenos básicos como la influencia de la personalidad o la emoción en los juicios morales (Bartels y Pizarro, 2011; Greene et al., 2001) y para la caracterización de poblaciones clínicas con dificultades de toma de decisiones sociales- incluyendo pacientes neurológicos, con trastornos por uso de sustancias o con trastornos de personalidad (Koenigs et al., 2007; Mendez, 2006; Van 't Wout y Sanfey, 2011; Volkow, Baler y Goldstein, 2011) en nuestro contexto. La disponibilidad de dos formas paralelas supone una ventaja adicional para su utilización como medida de cambio asociado a intervenciones que pueden modificar los mecanismos de toma de decisiones sociales, por ejemplo, en el caso de programas educativos o en el de intervenciones farmacológicas o neuroquirúrgicas. Por otro lado, la similitud entre los resultados americanos y españoles parecen indicar un fenómeno de transculturalidad en la toma de decisiones morales, apoyando así la teoría social-intuitiva de Haidt $(2001,2003)$ que propone que el juicio moral se da muy rápidamente, de forma relativamente automática e inconsciente, y la justificación racional del juicio -más permeable a las influencias culturales- se elabora a posteriori. Esta homogeneidad transcultural nos 
permite adoptar, en sus aspectos esenciales, el cuerpo teórico y la metodología utilizada en Estados Unidos.

Una de las asunciones centrales de este cuerpo teórico es el hecho de que los dilemas que contienen una mayor carga emocional (sobre todo los que requieren una intervención personal frente a una intervención instrumental o impersonal) tienden a generar menos respuestas utilitaristas. Nuestros resultados son congruentes con esta noción, ya que los dilemas personales generaron una proporción significativamente menor de respuestas afirmativas; es decir, ante tales situaciones hipotéticas, los sujetos rechazan llevar a cabo una acción emocionalmente muy aversiva (p.e., asfixiar a un bebé), pese a que esta renuncia conlleva el perjuicio de un número mucho mayor de personas. Adicionalmente, nuestros resultados mostraron que en los dilemas de autobeneficio -en los que la acción de matar se lleva a cabo para salvar la propia vida y la de los demás, y en los de muerte inevitable-en los que se mata a alguien que de todas formas iba a morir, los sujetos responden de forma más utilitarista. El primero de los sesgos es explicado por Moore et al. (2008) en términos de ventajismo evolutivo, que hace que tendamos a proteger de manera relativamente automática e intuitiva nuestra propia supervivencia. El segundo es atribuido a heurísticos de economía racional, que fomentan la tendencia a salvaguardar el mayor número posible de vidas una vez que la pérdida es inevitable (p.e., es el caso de los grupos de montañeros que deciden abandonar a un compañero en la cumbre -una vez descartada cualquier posibilidad de supervivencia, para salvar al grueso de la expedición). Los sesgos asociados a la dicotomía personal-impersonal $v$ s. pérdida evitable-inevitable se ubican en los dos extremos anticipados por la teoría del doble proceso: en el primer caso la carga emocional supera a la evaluación cognitiva y el juicio se decanta hacia la respuesta no-utilitaria, mientras que en la segunda dicotomía la carga emocional se diluye en beneficio del cálculo racional, favoreciendo la respuesta utilitarista. El sesgo asociado al autobeneficio estaría en consonancia con la teoría social-intuitiva (Haidt, 2001, 2003), favoreciendo el heurístico mejor engranado y automatizado evolutivamente -el de la supervivencia individual.

La estructura factorial encontrada inicialmente no se corresponde con las ocho categorías de dilemas propuestos por Moore. Este resultado no es sorprendente teniendo en cuenta que se trata de una propuesta con un alto grado de especificidad comparado con otros autores, que solo distinguen la existencia de dos grandes categorías (personal e impersonal). Si partimos del postulado de una única dimensión principal personal-impersonal (Greene et., al 2001; 2004), la estructura bifactorial que presentan los datos apoya, al menos parcialmente, la existencia de la dimensión, ya que en ambas formas del cuestionario más del 70\% de los ítems se agrupan en la categoría para la que fueron diseñados por el autor del cuestionario.

La inclusión en nuestro estudio de la nueva variable de dificultad de juicio introduce importantes implicaciones teóricas. En el caso de la tipología personal-impersonal las dos variables principales están alineadas -los dilemas personales generan más dificultad de juicio y, por tanto, menor tendencia a escoger respuestas utilitaristas. En cambio, en los otros dos tipos de dilemas (muerte inevitable y autobeneficio) el mayor número de respuestas utilitaristas se corresponde con una mayor dificultad de juicio. Estos resultados podrían explicarse por un sesgo normativo de aversión a la culpa, que comparte mecanismos neuropsicológicos con la activación emocional asociada a los dilemas morales (Chang et al., 2011); las respuestas afirmativas se asociarían en este caso a una mayor carga emocional vinculada al conflicto asociado a la culpa. En ambos resultados la variable "dificultad de juicio" se comporta como un índice discriminativo asociado a la carga emocional de los dilemas. Por tanto, nuestros resultados son congruentes con los numerosos hallazgos previos que destacan el papel de la emoción en los juicios morales (Greene et al., 2001, 2004; Koenigs et al., 2007; Luo y Blair, 2006; Moore et al., 2008; Oliveira-Souza et al., 2002). Aunque la escala de dificultad de juicio permite cumplir el objetivo de validar la distinción entre los tipos de dilemas, la distribución de las puntuaciones descriptivas parece indicar una falta de discriminación de las opciones de la escala debido a la inclusión de un número de respuestas demasiado elevado. Teniendo en cuenta estos resultados, para próximos estudios se propone la disminución del número de categorías, siendo según la psicología cognitiva entre $5 \pm 2$ el número idóneo.

Entre las limitaciones del estudio, es importante resaltar que estos resultados pertenecen a una muestra formada mayoritariamente por mujeres jóvenes de alto nivel educativo. Aunque los modelos imperantes defienden la universalidad de los juicios morales y nuestros resultados son muy similares a los obtenidos en la muestra estadounidense (de composición demográfica más heterogénea), sería recomendable ampliar estos resultados en muestras más representativas de la demografía española. Asimismo, futuros estudios deberían explorar la utilidad de esta herramienta en poblaciones clínicas, caracterizando los patrones de respuesta de distintos grupos psicopatológicos.

En conclusión, la adaptación española del cuestionario permite medir de manera fiable el juicio moral, postulándose como un instrumento potencialmente útil en la evaluación de poblaciones con problemas de toma de decisiones sociales y afectivas. 


\section{Referencias}

Anderson, S., Bechara, A., Damasio, H., Tranel, D. y Damasio, A. (1999). Impairment of social and moral behaviour related to early damage in human prefrontal cortex. Nature Neuroscience, 2, 1032-1037.

Bartels, D. M. y Pizarro, D. A. (2011). The mismeasure of morals: Antisocial personality traits predict utilitarian responses to moral dilemmas. Cognition, 121 (1), 154-61.

Blair, A., Mortoton, J., Leonard, A. y Blair, R. (2006). Impaired decisionmaking on the bais of both rexard and punishment information in individuals with psychopathy. Elsevier, 41, 155-165.

Bond, T. G. y Fox, C. M. (2007). Applying the Rasch model: fundamental measurement in the buman sciences. Routledge.

Casebeer, W. D. (2003). Moral cognition and its neural constituents. Nature Reviews Neuroscience, 4, 840-846.

Chang, L. J., Smith, A., Dufwenberg, M. y Sanfey, A. G. (2011). Triangulating the neural, psychological, and economic bases of guilt aversion. Neuron, 70, 560-572.

Cortés-Pascual, A. (2002) La contribución de la psicología ecológica al desarrollo moral. Un estudio con adolescentes, Anales de Psicologia, 18 (1), 111-134.

Fernández-Pinto, I., López-Pérez, B. y Márquez, M. (2008). Empatía: Medidas, teorías y aplicaciones en revisión. Anales de Psicología, 24(2), 284-298.

Fisher, W. J. (1992). Reliability Statistics. Rasch Measurement Transactions, 6(3), 238.

Goldberg, E. (2001). The executive brain: Frontal lobes and the civilized mind. New York: Oxford University Press.

Grande-García, I. (2009). Neurociencia social: El maridaje entre la psicología social y las neurociencias cognitivas. Revisión e introducción a una nueva disciplina. Anales de Psicología, 25(1), 1-2.

Greene, J. D., Sommerville, R. B., Nystrom, L. E., Darley, J. M. y Cohen, J. D. (2001). An fMRI investigation of emotional engagement in moral judgment. Science, 293, 2105-2108.

Greene, J. D. y Haidt, J. How (and where) does moral judgment work? (2002). Trends in Cognitive Sciences, 6, 517-23.

Greene, J. D. (2003). From neural 'is' to moral 'ought': what are the moral implications of neuroscientific moral psychology? Nature Reviews Neuroscience, 4, 846-849.

Greene, J. D., Nystrom, L. E., Engell, A. D., Darley, J. M. y Cohen, J. D. (2004). The neural bases of cognitive conflict and control in moral judgment. Neuron, 44, 389-40.

Greene, J. D. (2007) Why are VMPFC patients more utilitarian? A dualprocess theory of moral jugment explain. Trends in Cognitive Sciences, 11(8), 322-323.

Haidt, J. (2001). The emotional dog and its rational tail: A social intuitionist approach to moral Judgment. Psychological Review, 108(7), 804-834.

Haidt, J., Rosenberg, E. y Hom, H. (2003). Differentiating diversities: moral diversity is not like other kinds. Journal of Applied Social Psychology, 33, 136.

Hauser, M., Cushman, F., Young, L., Jin, R. y Mikhail, J. (2007). A dissociation between moral judgments and justifications. Mind \& Language, 22, $1-21$

Heekeren, H. R., Wartenburger, I., Schmidt, H., Schwintowski, H. P. y Villringer, A. (2005). Influence of bodily harm on neural correlates of semantic and moral decision-making. Neuroimage, 24, 887-897.

Kahane, G. y Shackel, N. (2010). Methodological Issues in the Neuroscience of Moral Judgement. Mind \& language, 25(5), 561-582.

Koenigs, M., Young, L., Adolphs, R., Tranel, D., Hauser, M. y Damasio, A. (2007). Damage to the prefrontal cortex increases utilitarian moral judgements. Nature, 447(7138), 808-911.

Luo, Q. y Blair, R. (2006). The neural basis of implicit moral attitude-and IAT study using event-related FMRI. Neuroimage, 30, 1449-1457.
McGuire, J., Langdon, R., Coltheart, M. y Mackenzie, C. (2009). A reanalysis of the personal/impersonal distinction in moral psychology research. Journal of Experimental Social Psychology, 45, 577-58.

Mendez, M. F. (2006). What frontotemporal dementia reveals about the neurobiological basis of morality. Medical Hypotheses, 67(2), 411-418.

Moll, J., Oliveira-Souza, R., Bramati, I. E. y Grafman, J. (2002). Functional networks in emotional moral and nonmoral social judgments. Neuroimage 16, 696-703.

Moll, J., Oliverira-Souza, R., Moll, F. T. e Ignacio, F. A. (2005). The moral affiliations of disgust: a functional MRI study. Cognitive and Behavioral Neurology, 18, 68-78.

Moll, J., Zahn, R., Oliveira-Souza, R., Kruger, I. y Grafman, J. (2005). Opinion: The neural basis of human moral cognition. Nature Reviews Neuroscience, 6, 799-809.

Moore, A., Clark, B. y Kane, M. (2008). Who Shalt Not Kill? Individual Differences in Working Memory Capacity, Executive Control and Moral Judgment. Psychological science, 6(19), 549-557.

Nosek, B., Banaji, M. y Greenwald, A. (2002). Harvesting implicit group attitudes and beliefs from a demonstration website. Group Dynamics, 6 (1), 101-115.

Oliveira-Souza, Bramati, I. y Grafman J. (2002). Functional networds in emocional moral and nonmoral social judgements. Neuroimage, 16, 696703.

Preston, S., Bechara, A., Damasio, H., Grabowski, T., Stansfield, R., Mehta, S. y Damasio, A. (2007), The Neural Substrates of Cognitive Empathy. Social Neuroscience, 2(3-4), 254-275.

Rilling, J. K y Sanfey, A. G. (2011). The neuroscience of social decisionmaking. Annual Reviews of Psychology, 62, 23-48.

Sánchez-Navarro, J. P y Román, F. (2004). Amígdala, corteza prefrontal y especialización hemisférica en la experiencia y expresión emocional. Anales de Psicologia, 20(2), 223-24.

Smith, R. M., y Miao, C. Y. (1994). Assessing unidimensionality for Rasch measurement. Objective Measurement: Theory into Practice (Wilson, M., Vol. 2, pp. 316-327). Norwood NJ: Ablex.

Tennant, A. y Conaghan, P. G. (2007). The Rasch measurement model in rheumatology: What is it and why use it? When should it be applied, and what should one look for in a Rasch paper? Arthritis Care and Research, 57(8), 1358-1362.

Tennant, A. y Pallant, J. F. (2006). Unidimensionality Matters! Rasch Measurement Transactions, 20(1), 1048-51.

Tirapu-Ustárroz, J., Pérez-Sayez, G., Erekatxo-Bilbao, M. y Pelegrín-Valero, C. (2007). ¿Qué es la teoría de la mente? Revista de Neurología, 44(8), 479489.

Van't Wout, M. y Sanfey, A. G. (2011). Interactive decision-making in people with schizotypal traits: A game theory approach. Psychiatry Research, 185 (1), 92-96.

Vélez, A., Chayo-Dichi, R., Arias, N., Castillo, G. y Ostrosky-Solís, F. (2003). Emociones morales: Una batería para su medición. Revista Neuropsicologia, Neuropsiquatría y Neurociencias, 5, 189-199.

Verdejo-García, A. y Bechara, A. (2010). Neuropsicología de las funciones ejecutivas. Psicothema, 2(22), 227-235.

Volkow, N. D., Baler R. D. y Goldstein, R. Z. (2011). Addiction: pulling at the neural threads of social behaviors. Neuron, 69(4), 599-602.

Young, L., Cushman, F., Hauser, M. y Saxe, R. (2007). The neural basis of the interaction between theory of mind and moral judgment. PNAS, $104(20), 8235-824$.

Young, L. y Koenigs, M. (2007). Investigation emotion in moral cognition: a review of evidence form functional neuroimaging and neurpsychology. British Medical Bulletin, 84, 69-79.

(Articulo recibido: 11-10-2011; revisado: 15-10-2012; aceptado: 15-10-2012) 